\title{
Effectiveness and efficiency under competition: the Cochrane test
}

\author{
Donald W Light
}

Division of Social

Behavioural Medicine, University of Medicine and Dentistry of New Jersey, New Jersey 08103, USA

Donald W Light, PHD, professor

BMY 1991;303:1253-4
A major purpose of introducing economic competition into health services in Great Britain, Holland, Germany, and elsewhere is to increase efficiency and cost effectiveness. Paradoxically, competitive markets may actually reduce efficiency because provider-sellers dominate the market ${ }^{1}$ and may respond in ways that decrease efficiency ${ }^{2}$ and because many inefficiencies are embedded in "the way things are done." 3 There are more direct routes to efficiency and cost effectiveness that avoid the distortions and added costs of markets. Several insights can be gleaned by revisiting Archie Cochrane's classic work, Effectiveness and Efficiency. ${ }^{+}$

Cochrane's message is the primacy of effectiveness for attaining efficiency. The current focus on managerial efficiency in the NHS would seem to him misdirected. How, he might ask, can one increase efficiency through competitive contracts if the contractors do not know what is effective?

Cochrane is more clearheaded than almost anyone concerned with outcomes (effectiveness) and efficiency in professional circles today. How many outcome studies can pass what might be called the Cochrane test?

\section{(1) Consider anything that works}

Cochrane tells of a doctor who saw a table from a clinical trial of coronary care units that showed that more of those treated at home died than those treated in the coronary care unit. He immediately declared the study unethical and demanded that the trial be stopped. Actually the figures had been reversed. When Cochrane showed the doctor the correct data the doctor refused to declare the trial unethical and demand that patients be stopped from going to coronary care units.

How many of us are willing to do more than compare alternative medical interventions and see whether a medical treatment does more harm than no intervention? Consider all the money spent on lower back problems with little evidence that many treatments do more good than bed rest and pain killers, exercises, or manual manipulation.

This first test relates especially to psychiatry, one tenth of the NHS budget and growing. How effective is treatment for mild depression and anxiety compared with getting a pet, praying, or joining a mutual support group? Are psychiatrists any more effective than psychiatric social workers and nurses in treating the more common disorders?

In sum, the first test is how open is evaluation research to measuring the relative effectiveness of any reasonable alternative-whether inside established medicine or not?

Current policies focused on inefficiencies overlook what medical services are supposed to do. Efficiency is not measured in terms of number of treatable cases treated or preventable disorders prevented or pain and suffering relieved. Yet without such measures, not funding treatable cases becomes efficient by saving money. We need to find a way to "charge" a health care system for each day of suffering and dysfunction experienced by treatable and preventable cases Otherwise, fewer services are the obvious solution to budgetary pressures, especially for those less able to voice their objection.

\section{(3) Minimise ill timed interventions}

How many outcome studies use timing as a serious variable? The point at which an intervention occurs is for Cochrane an important dimension of effectiveness. For example, Joseph Gonnella, the dean of Jefferson Medical College in Philadelphia, has spent the past decade developing one of the best models of disease staging. Recently he applied it to hospital admissions and found that one fifth of patients were admitted later than would be optimal. As a result they stayed twice as long, cost three times as much, and got better less often than a matched sample of patients admitted at the right time. ${ }^{5}$

Effective and timely treatments should be linked to waiting lists. Putting off the treatment of problems that may go away or treatments whose timing and effectiveness still need to be established would be the most cost effective focus for a policy of waiting. As it is now, many countries pay for such treatments while patients wait for non-urgent but effective treatments

\section{(4) Treat patients in the most cost effective place}

Almost 20 years before the Audit Commission's report on the underuse of day surgery,${ }^{6}$ Cochrane cited clinical trials dating back to 1947 that showed that patients with hernias did as well with day surgery as with an average inpatient stay of $9 \cdot 1$ days. Imagine the money saved and the beds made available to others had most hernia operations since 1972, or even since 1947 , been done on a day basis.

What does it take to get surgeons and physicians to adopt the most cost effective practices in less than 40 50 years? Apparently, tight budgets did not do the job, and the budgetary pressures of competitive contracts alone may not reach such embedded inefficiencies either. ${ }^{3}$ The record of doctors persisting in costly, il timed, or ineffective practices is the principal reason why governments and other payers are now trying to make them accountable through outcome research, protocols, and medical audit.

\section{(2) Make effective treatments available to all}

The second part of the Cochrane test looks at the other side: Are effective treatments available free to everyone who can benefit? If not, then the health care system is by that degree ineffective at the macro level in its primary goal and inefficient in treating the ills of society.

\section{(5) Prevent only what is preventable}

The Cochrane test applies with equal force and practicality to prevention. One should spend money only on preventive measures that are more effective 


\section{The Cochrane test}

(1) Consider anything that works

(2) Make effective treatments available to all

(3) Minimise ill timed interventions

(4) Treat patients in the most cost effective place

(5) Prevent only what is preventable

(6) Diagnose only if treatable

than no treatment or alternative treatments, and one should screen only for treatable or preventable problems. In 1972 Cochrane called cervical smears "the saddest sin of commission in the NHS." (The worst sins of omission were antismoking programmes and preventing unwanted pregnancies.) The incidence of cervical cancer was rare and it was still more rare to find at treatable stage, Cochrane argued, and its death rate fell at the same rate before mass screening began as after.

Cochrane would have shaken his head in disbelief at the way prevention protocols in the new general practitioner contract make the government pay for measures well beyond what the evidence shows to be reasonable. ${ }^{6}$

\section{(6) Diagnose only if treatable}

Cochrane understood 20 years ago that doctors like to make diagnoses. Accurate, even insightful, diagnosis has long earned the profession respect, even if nothing can be done. Soon, advances in microbiology and genetics will enable us to identify disorders and predict them before they happen, far beyond what we can treat. Outside research, Cochrane thought such diagnostics were a professional indulgence that signals to the patient that effective treatment will follow when it may not.

\section{How the NHS scores}

How does the NHS or any other system score on the Cochrane test?

The NHS would come out fairly well on items 2 (making treatments available) and 6 (diagnosing only treatable disorders), better than many other systems, but it would come out worse on 4 (treat in the most cost effective place) and 5 (prevent only preventable conditions) and poorly on items 1 (test effectiveness) and 3 (test effectiveness at different times). So would almost every other system. In short, virtually every health care system in the world has a long way to go in meeting these six criteria for cost effective care.

It makes little sense for a health care system to become preoccupied with managerial efficiency if it does not first determine which services are worth providing, and where and when, and which services should be dropped. Once a health care system scores well on the Cochrane test most of its inefficiencies will be eliminated. This is precisely what needs based budgeting and purchasing can accomplish. The Cochrane test provides guidelines for health authorities and directors of public health as they take up this rare opportunity to allocate funds wisely by measuring the levels of dysfunction and pain in the population and then purchasing the services that most effectively reduce them.

I thank A W Macara, consultant in public health medicine, University of Bristol, for his careful review and suggestions on an earlier draft. 1 Light DW. Learning from their mistakes? Health Service fournal 1990;5221:
1470-2.

2 Light DW. Bending the rules. Health Service fournal 1990;5222:1513-5.

3 Light DW. Embedded inefficiencies. Lancet (in press).

4 Cochrane A. Effectiveness and efficiency: random reflections on health services. London: Nuffield Provincial Hospitals Trust, 1972.

5 Gonnella JS, Louis DZ, Zeleznik C, Turner BJ. The problem of late hospitalization: a quality and cost issue. Academic Medicine 1990;65:314-9. 6 Audit Commission. A short cut to better services: day surgery in England and Wales. London: HMSO, 1990.

(Accepted 14 October 1991)

\title{
Health and the Environment
}

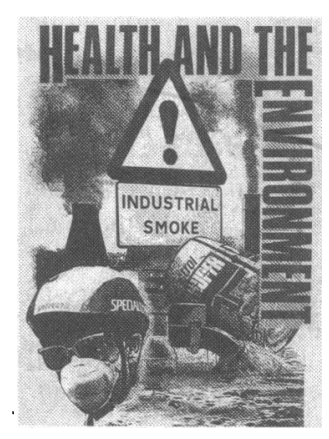

\section{Health implications of climatic change}

\author{
Fiona Godlee
}

Every age has its catastrophe theory. In the past decade alone scientists have threatened us with a new ice age and a nuclear winter. Now two new threats confront us, global warming and the destruction of the ozone layer, linked by their origin in man's pollution of the environment. Both have enormous implications for health. This article will examine the potential impacts of global warming on health. Next week's article will examine the dangers of ozone depletion.

\section{Global warming}

Without some form of insulating layer to trap heat from the sun the earth could not sustain life. Its surface temperature would be $40 \mathrm{~K}$ lower and the oceans would freeze.' Insulation, largely in the form of atmospheric carbon dioxide, allows the passage of visible and ultraviolet light from the sun while preventing too much of the solar heat that radiates from the earth's surface from escaping. But the amount of carbon dioxide and other insulating, or greenhouse, gases in the atmosphere is now rising fast, with potentially damaging effects on the earth's climate and on human health.

Carbon dioxide is produced by animals and plants and the burning of fossil fuels. Because trees fix carbon from the air, levels of carbon dioxide are also increased by deforestation. Each year 17 million hectares of tropical forest are being destroyed - an area the size of Austria. ${ }^{2}$ In the past hundred years, since the beginning of the industrial revolution, the concentration of carbon dioxide in the atmosphere has increased by a quarter and is currently increasing at a rate of about $0.5 \%$ a year. ${ }^{3}$ Concentrations are now higher than at any time in the past 16000 years. ${ }^{4}$ In 1988 the world produced five billion tonnes of carbon dioxide, and the atmospheric concentration was estimated at 352 parts per million. ' If in the next 100 years consumption of fossil fuels doubles - a conservative estimate-the concentration may rise to 600 parts per million: twice the level in $1850 .^{5}$

In terms of sheer bulk atmospheric carbon dioxide is the main contributor to the greenhouse effect. But other gases present in the atmosphere-methane, 